\title{
Information Theory and Natural Selection
}

\author{
Ryota Morimoto*
}

\begin{abstract}
Recently philosophers of biology have debated over the status of evolutionary theory; is it interpreted as realistic or anti-realistic? I discuss this issue by using Fisher's formula of natural selection as an example. In generalizing evolutionary phenomena, we need only partial information. So I compare the formula of natural selection with information theory, which gives us a reasonable tool of inference on the basis of partial information, and show that the formula of natural selection may be regarded as a part of information theory. I conclude that there is the mixture of realistic and anti-realistic factors in the formula of natural selection, and that the aim of evolutionary theory is rational prediction or explanation of phenomena on the basis of partial information, not complete description of it on the basis of full information.
\end{abstract}

\section{Introduction}

The famous biologist, T. Dobzhansky (1973) once said, "Nothing in biology makes sense except in the light of evolution." Apart from the truth of this statement, it is certain that evolutionary theory is important in biology and informs us much about the nature of life. For example, the different species are descended from a common ancestor and have evolved by natural selection. In addition, DNA molecules are base unit of the heredity and are translated to amino acids. Modern evolutionary theory has brought new understanding about the nature of life.

In spite of such situations, some philosophers of biology doubt the reality of evolutionary theory. ${ }^{1}$ A source of this doubt is the probability concept in evolutionary theory. Since our worldview is based on Newtonian mechanics, which is a non-probabilistic or deterministic theory, we have the deterministic worldview as a common sense. And then, there has been thought that the probabilities appeared in the scientific context is interpreted as our ignorance of the deterministic world; i.e. they don't represent the real world. Modern evolutionary theory, however, is indispensable with the probability concept. Recently philosophers of biology have debated over the status of evolutionary theory; is it interpreted as realistic or antirealistic? ${ }^{2}$ Some philosophers have argued it is interpreted as realistic (see, e.g., Sober

* Keio University. e-mail: ryota@pb3.so-net.ne.jp

${ }^{1}$ See Rosenberg (1994), Horan (1994), Graves, Horan and Rosenberg (1999).

${ }^{2}$ Usually discussions of scientific realism are about physical theory. But the issue con- 
1984. Brandon and Carson 1999, and Stamos 2000). Others, on the contrary, have argued it is anti-realistic (see, e.g., Rosenberg 1994, Horan 1994, Graves, Horan and Rosenberg 1999). In this paper I will discuss this issue by using Fisher's formula of natural selection as an example. In the traditional debate, the notions of realistic and anti-realistic are formulated as follows. If theory won't be recast with increasing our knowledge or information, theory is interpreted as realistic. Otherwise, it is interpreted as anti-realistic. ${ }^{3}$ I adopt these formulations here.

In this paper, first I survey the two opposite characterizations of evolutionary theory; one is based on Newtonian mechanical point of view and the other is based on quantum mechanical point of view. And I indicate some similarities between them (section 2). Second, I criticize both characterizations and introduce the significant principle of information theory called, 'maximum entropy principle' by which we can utilize partial information (section 3). Third, I show that the principle of information theory can be applied to that of Fisher's formula of natural selection and make a comparison between them (section 4). Fourth, I discuss Fisher's formula of natural selection from information theoretical point of view and show that the formula of natural selection may be regarded as a part of information theory (section 5). Lastly, I conclude that there is the mixture of realistic and anti-realistic factors in evolutionary theory including Fisher's formula of natural selection, and that the aim of evolutionary theory is rational prediction or explanation of phenomena on the basis of partial information, not complete description of it on the basis of full information (section 6).

\section{Bipolarization}

The mathematical approaches of evolutionary theory began at the $20^{\text {th }}$ century and many evolutionary formulas have been made. R. Fisher presented the most famous formula of natural selection in his book The Genetical Theory of Natural Selection (1930). The formula he himself called 'the fundamental theorem of natural selection' has been believed to be valid. According to his formula, when natural selection works at the biological population, the change of the average fitness $\bar{w}$ in the population is expressed

$$
\frac{d \bar{w}}{d t}=\sum p_{i}\left(w_{i}-\bar{w}\right)^{2}
$$

where $p_{i}$ is the frequency of the allele $i$ in the population and $w_{i}$ is the fitness of that allele. This equation indicates that "(the) rate of increase in fitness of any organism at any time is equal to its genetic variance in fitness at that time." (p.35; italic in

sidered here is about evolutionary theory, not about physical theory.

${ }^{3}$ See Rosenberg (1994 p.7), and Brandon and Carson (1996 p.316). 
original) Namely the more diverse the biological population is, the fitter it is. Since this formula represents properties of population or of aggregate of organisms, the probability concept is necessary for its formula. ${ }^{4}$ And then, the probability concept appears in Fisher's formula as well as any other formulas of natural selection. ${ }^{5}$

Although these formulas can explain or predict the phenomena successfully, the probability concept is integral part of evolutionary theory including the formulas of natural selection. What dose the probability concept represent? If it represents the reality of biological world, then it means that the world is probabilistic or indeterministic. If not, it merely reffects our ignorance of the deterministic world. The problem considered here is 'is evolutionary theory interpreted as realistic or anti-realistic with respect to the usage of probability concept?'

There propose two opposite characterizations of evolutionary theory. One is based on Newtonian mechanical worldview and the other based on quantum mechanical worldview. These propose the bipolarized characterizations; the former is epistemic and the latter realistic with respect to evolutionary theory. ${ }^{6}$ I surveyed them elsewhere (Morimoto 2007b) and then I will sketch them briefly. From Newtonian mechanical point of view, the world is deterministic and the probability concept merely reflects our ignorance of the world state. From quantum mechanical point of view, on the contrary, the world is indeterministic and the probability concept represents indeterminacy of the real world.

To begin with, we will sketch the anti-realists' characterizations of evolutionary theory based on the Newtonian mechanical point of view. The anti-realists presuppose that evolutionary theory should basically represent an organism, i.e. a single macroscopic object. Since a single macroscopic object obeys by Newtonian mechanics, this position founds evolutionary theory on Newtonian mechanical point of view. ${ }^{7}$ According to a standard interpretation of Newtonian mechanics, a macroscopic object including a biological organism moves deterministically. And then, to the anti-realists, the evolutionary phenomena have to be deterministic. Thus there would be no need for the concept of probability in explaining evolutionary phenomena if we had com-

${ }^{4}$ The main subject of evolutionary theory is a biological population. Mayr (1976) called this way of thinking 'population thinking', and said as follows. "Darwin introduced into the scientific literature a new way of thinking, 'population thinking'." (p.410) For further discussion, see Morimoto (2007a).

${ }^{5}$ See Wright (1931) and Price (1970).

${ }^{6}$ Note again that realists/anti-realists here mean the persons who claim evolutionary theory, not physical theory, is interpreted as realistic/anti-realistic. In this controversy, it is assumed that physical and chemical theories are realistic. See also Rosenberg (1994 ch.1).

${ }^{7}$ Graves, Horan and Rosenberg present other reason why evolutionary theory should be founded on Newtonian mechanics. They claim, "The 'positing' of deterministic hidden variables in evolutionary theory has been essential to such theoretical advance" (1999 p.153). 
plete knowledge or full information. But indeed we finite creatures don't have full information. Hence we need the concept of probability in evolutionary theory. The probability concept used in modern evolutionary theory is supposed to our incomplete knowledge or our ignorance of biological world. That is, the interpretation of probability in evolutionary theory is anti-realistic. If we would come to know everything in future, then we could establish the non-probabilistic evolutionary theory by which the reality of deterministic biological world would be represented. Newtonian mechanical point of view is led to the conclusion that modern evolutionary theory is interpreted as anti-realistic.

Next, we will turn to the realists' characterization of evolutionary theory based on quantum mechanics. For all this argument is similar in framework to the one based on Newtonian mechanics, the conclusions are quite the opposite. The realists presuppose that evolutionary phenomena should be reducibly represented by a point mutation; i.e. a chemical reaction of a single DNA molecule. Since a point mutation of a DNA is a microscopic phenomenon, the realists found evolutionary theory on quantum mechanics. According to a standard (or classical) interpretation of quantum mechanics, i.e. Copenhagen interpretation, even if we had complete knowledge, a motion of a microscopic object had to be described probabilistically or indeterministically. A DNA molecule is a microscopic object, and then a point mutation has to be an indeterministic phenomenon. The realists, moreover, claim that indeterminacy at micro level may percolate up to the macro level phenomena. ${ }^{8}$ They argue as follows. There is a situation in which a point mutation will shift biological population from equilibrium state to nonequilibrium one. In this situation even a point mutation at micro level can effect the composition of the population at macro level. ${ }^{9}$ Thus they maintain that evolutionary phenomena at macro level are indeterministic and that interpretation of the probability concept used in evolutionary theory is realistic. Quantum mechanical point of view is led to the conclusion that evolutionary theory is realistic.

Two positions above characterize evolutionary theory quite the opposite, but I guess there are some similarities between these characterizations. (1) Both positions presuppose complete knowledge or full information. From Newtonian or quantum mechanical point of view, there is the issue whether the probability concept is necessary for an omniscient being. (2) Both positions characterize not biological but physical properties. Their viewpoints base on physical theories; Newtonian mechanics or

${ }^{8}$ Copenhagen interpretation of quantum mechanics claims that while phenomena at micro level are indeterministic, phenomena at macro level are deterministic. On the contrary, the position based on quantum mechanics here claims that phenomena are deterministic not only at micro level but also at macro level. This claim seems against Copenhagen interpretation.

${ }^{9}$ For further discussion, see Stamos (2000) and Glamour (2001). 
quantum mechanics. Even though there are some formulas of evolution, they pay little attention to these formulas. I criticize these two similarities in next section. ${ }^{10}$

\section{Maximum entropy principle}

Population evolves by means of natural selection when it contains heritable variation in fitness (Lewontin 1970). Fitness refers to an organism's or a trait's ability to survive or reproduce. In formulas of natural selection the fitness is a property of traits and is expressed quantitatively with the probability concept. Traits of organisms must inherit from ancestor whereas stones or balls don't. Heritability is an essential property of biological object while physics is indifferent to such a property. For example, physics treats a zebra's speed and a stone's speed in the same way. Physics alone can't distinguish heritable properties from non-heritable properties. Thus both characterizations based on physical theories (similarity (2) in section 2) miss the crucial property of biological object.

When we express fitness numerically in the formula of natural selection, it is necessary to specify the heritable trait in the population. For example, Drosophila has colored eyes, wings, legs, transverse black rings across their abdomen, and so on. ${ }^{11}$ And it is said that the numbers of Drosophila's genome are about 170 million base pairs. All these traits and genome are inherited. Even a single organism has a lot of traits and vast amounts of genome, much more a population. If we took full information into account, we couldn't generalize evolutionary phenomena at population level. Thus when we explain the evolution of Drosophila's colored eye, we will specify only this trait and discard information about many other properties. Even if we had full information or complete knowledge, we would discard almost all of them. In generalizing evolutionary phenomena at population level, we need only partial information against to full information. Thus full information or complete knowledge is not necessary for the formulas of natural selection. Note that partial information doesn't mean incomplete, since we would discard almost all of them even if we had complete information. Hence partial information represented biological properties is sufficient for evolutionary theory. Hence similarity (1) in section 2 is unnecessary.

Information theory gives us a reasonable tool of inference on the basis of partial information. In the reminder of this section I consider one significant tool used

${ }^{10}$ I have discussed and criticized other similar features between both positions. They presuppose that evolutionary theory should be represented by a single object, i.e. a single organism or a single DNA molecule. Moreover they presuppose that evolutionary theory can be reduced to physical theory. For further discussion about these features, see Morimoto (2007b).

${ }^{11}$ Again, note that physics alone can't explain the difference between heritable properties like colored eyes or wings, and non-heritable properties like the ink biologists marked. 
in information theory and in next section I apply it to Fisher's formula of natural selection. Information theory was started from C. Shannon's seminal paper "A Mathematical Theory of Communication." (1948) He said, "The fundamental problem of communication is that of reproducing at one point either exactly or approximately a message selected at another point." (p.379) To satisfy this purpose, he introduced information entropy, rate of transmission, channel capacity, and so on. In order to transmit reliable information over a noisy channel, it is necessary to reduce and correct the errors, to compress the messages, or to increase the rate of transmission. Information transmits the most efficiently when the defined quantities like the information entropy or the rate of transmission have maximum values. Such extrema can be derived from 'maximum entropy principle', which was named by statistical physicist, E.T. Jaynes. ${ }^{12}$

Let us consider the principle by using an example. We will throw a dice and get money $f_{i}$ yen of 10000 times of the spot $i$. And we don't know the probability $p_{i}$ of getting $i$. In using maximum entropy principle, we can derive $p_{i}$ from maximizing the information entropy subject to partial information in hand. In this case, we have two kinds of information; (1) probability satisfies the normalized condition which is one of the Kolmogorov axioms of probability, and (2) the expectation of getting money is given. These kinds of information are formalized as

$$
\sum_{i} p_{i}=1
$$

and

$$
\bar{f}=\sum_{i} p_{i} f_{i}
$$

where $\bar{f}$ stands for the expectation. In addition, Shannon proved that the quantity, which is positive, which increases according to the increasing uncertainty, and which is additive for independent source of uncertainty, has to be the information entropy $H$

$$
H=-\sum_{i} p_{i} \log p_{i}
$$

In deriving $p_{i}$ on the basis of partial information, we ought to use the probability which has maximum entropy subject to whatever we know. This is the only reasonable tool we can use. To maximize equation (3.3) subject to constrains (3.1) and (3.2), we introduce Lagrangian multipliers $\alpha$ and $\beta$ and define the function $L$

$$
L=H+\alpha\left(\sum_{i} p_{i} f_{i}-\bar{f}\right)+\beta\left(\sum_{i} p_{i}-1\right) .
$$

\footnotetext{
12 See Jaynes (1957a).
} 
The first term in the right hand side of equation (3.4) is the objective function. The second and the last terms are the constraints. Information entropy $H$ is maximized under these constraints only if function $L$ has the maximum value. Thus from finding the extrema of function $L$, we can derive $p_{i}$ which has maximum entropy

$$
p_{i}=\frac{\exp \left(\alpha f_{i}\right)}{\sum_{i} \exp \left(\alpha f_{i}\right)},
$$

where $\alpha$ can be deduced by substituting equation (3.5) to (3.2). ${ }^{13}$ This is maximum entropy principle, which is an optimization tool and is called Lagrange multipliers in mathematics. In the case above we maximize entropy function. In other case we can maximize other function depending on our purpose. For example, if we need to reduce errors, we maximize the function called, "generalization error function." By using the principle of information theory, we can utilize partial information most efficiently.

\section{Application to Fisher's formula of natural selection}

Jaynes, who introduced 'maximum entropy principle' in statistical physics, said, "We suggest that the maximum-entropy formalism provides the general solution to the problem of 'setting up an ensemble' to describe an arbitrary macroscopic situation." (1963 p.206) Evolutionary theory explains or predicts macroscopic phenomena of biological population, i.e. of ensemble of organisms. And I have argued in section 3 that partial information is sufficient for evolutionary theory. Therefore we may expect evolutionary theory can be derived by means of maximum entropy principle. In this section, I show that it also can be applied to Fisher's formula of natural selection.

Let us return to Fisher's formula of natural selection. Equation (2.1) represents the change of the average fitness equals to the variance of the population. His formula, however, doesn't explain or predict the change in gene frequency $d p_{i} / d t$. Thus M. Kimura derived the change in gene frequency $p_{i}$ under the same conditions as Fisher's formula. ${ }^{14}$ In order to derive such a formula, we just know at least two kinds of information; (1) probability $p_{i}$ satisfies the normalized condition, which is one of the axioms of probability, and (2) the average fitness $\bar{w}$ is defined. These kinds of information are formalized as

$$
\sum_{i} p_{i}=1
$$

and

${ }^{13}$ For more detail derivation, see Appendix 1.

14 See Kimura (1958), and Crow and Kimura (1970). 


$$
\bar{w}=\sum_{i} p_{i} w_{i}
$$

where $w_{i}$ is the fitness of allele $i$. Just from these kinds of information, we can derive not only Fisher's formula but also the change in gene frequency by means of natural selection. Now let us derive the change in gene frequency when the average fitness will change most. In deriving $d p_{i} / d t$ on the basis of partial information, we ought to use the probability which has maximum change of the average fitness subject to whatever we know. To maximize the change of the average fitness subject to constrains (4.1) and (4.2), we again introduce Lagrangian multipliers $\alpha^{\prime}$ and $\beta^{\prime}$ and define the function $L^{\prime}$

$$
L^{\prime}=\frac{d \bar{w}}{d t}+\alpha^{\prime}\left(\sum_{i} p_{i} w_{i}-\bar{w}\right)+\beta^{\prime}\left(\sum_{i} p_{i}-1\right) .
$$

The first term in the right hand side of equation (4.3) is the objective function. The second and the last terms are the constraints. The change of the average fitness $d \bar{w} / d t$ is maximized under these constraints only if function $L^{\prime}$ has the maximum value. Thus from finding the extrema of function $L^{\prime}$, we can derive the change of gene frequency $d p_{i} / d t$ in the maximum change of the average fitness

$$
\frac{d p_{i}}{d t}=p_{i}\left(w_{i}-\bar{w}\right)
$$

Kimura worked this out and called the derivation principle 'maximum principle of natural selection.' ${ }^{15}$ He introduced it not because he empathized similarity of Fisher's formula of natural selection and information theory, but because Fisher's formula "paid little attention to how natural selection changes gene frequencies." (1958 p.157) Indeed Kimura didn't mention information theory at all. Now I compare Fisher's formula of natural selection with information theory and demonstrate the similarities between them.

First, we find one similarity in constraints of information theory and of the formula of natural selection. Equations (3.1) and (3.2) are constraints in information theory, and equations (4.1) and (4.2) in the formula of natural selection. Equation (3.1) is the same as (4.1) that is one of the axioms of probability and then is an indispensable condition to use probability. Equations (3.2) and (4.2) are the expectations of the functions and are mathematically equivalent. The expectations of the rate of transmission or of getting money are defined in information theory, and the average fitness is defined in the formula of natural selection. The defined functions vary with our purposes or interests. If we intend to construct the channel of efficient transmission, we define the rate of transmission. If we want to know how much we

\footnotetext{
${ }^{15}$ For more detail deviation, see Appendix 2.
} 
will get in gambling, we define the expectation of getting money. If we explain the change of gene frequency by means of natural selection, we define the average fitness. In this way we define the functions depending on the context while the functions are mathematically equivalent.

Second, we find another similarity in the derivation. In information theory, maximum entropy principle is used to transmit information most efficiently. In the formula of natural selection, maximum principle of natural selection is used to predict or explain the evolutionary phenomena. Mathematically both principles are the same tool called Lagrangian multipliers. Thus Fisher's formula of natural selection is similar to information theory in not only constraints but also the derivation principle. Lagrangian multipliers subject to constraints may be interpreted as a rational principle subject to partial information. Thus we may consider both principles of information theory and the formula of natural selection as rational tools to utilize partially given information. Therefore Fisher's formula of natural selection may be thought to be based on partial information, and may be regarded as a part of information theory.

\section{From information theoretical point of view}

Information theory provides us a rational principle to utilize partially given information most efficiently. Maximum entropy principle is one instance. Information theory is not aimed for tracing time evolution of information or describing a dice's trajectory. Rather, information theory has advantages of speedy and correct transmission of information, prediction of dicing, or explanation of the outcomes, etc. That is to say, the aim of information theory is not complete description of phenomena but prediction or explanation subject to partial information. This point is similar in the formula of natural selection. As I have said in section 3, complete knowledge or full information isn't necessary for the formula of natural selection. Fisher's formula is not aimed for complete description of phenomena. Rather, it can rationally predict or explain the change in gene frequency on the basis of partial information. Information theory and the formula of natural selection have advantages to utilize partial information.

And the amount of information is relevant to the accuracy of prediction. The more information we have, the more accurate prediction we can make. For example, we have already known that the dice has 6 faces. In addition, we acquire information that a 3 was rolled 50 times in a sequence of 100 rolls. Then we can predict the outcomes more accurately by associating the additional useful information. In case of evolutionary theory, if we know only the axioms of probability and the definition of the average fitness, we can predict the change in gene frequency by means of natural selection. In additions, if we get information about the effect of genetic factor like mutation, epistasis, and so on, we can predict the change more accurately. And if 
we get additional information about the effect of environment factor, we can predict it more accurately. ${ }^{16}$ Not only the accuracy of prediction but also the probability in evolutionary theory varies with the amount of useful information. This means that the probability used in evolutionary theory is interpreted as anti-realistic if we follow the traditional expressions. From information theoretical point of view, the accuracy of prediction can be regarded as the amount of useful information. And the probability concept in evolutionary theory implies the epistemic factors in the sense that it varies with the amount of information we have.

Moreover, even if we don't have much information, we can explain the change in the population although we predict it less accurately. For example, when we consider information only about natural selection without attending to the effect of genetic factor, we can explain how the population will evolve under natural selection. On the contrary if we take information about genetic factor or environmental factor into account, we can't explain the effect of natural selection on the population although we can explain the net effect of evolutionary factors. Thus depending on our purpose, we will change the amount of information to consider. It is not always defective but is sometimes advantageous to decrease the amount of information. Note that the amount of information isn't always correlated with explanation of the reality. For example when we explain the revolution of the earth, we don't usually take information about my height or speed into account. Such information is useless for explanation even though it may be helpful to predict the orbit of earth more accurately. In explaining it, we need information not about my height or speed, but about the mass of sun and earth, the distance between them, their velocities and so on. Even if I get fat, it is the case that the earth still revolves. Even if I get fat and you don't know about that, you can explain the revolution of the earth. And such explanation may capture some aspects of reality. In the same way, we can explain the change in Drosophila's colored eyes' frequency without taking the color of Dobzhansky's eyes into account, and such an explanation may capture partial reality. Hence even if we don't take full information into account or we discard any information, we can still explain some aspects of reality. And in this sense the probability concept used in evolutionary theory implies objective factors.

Let us return to the problem, 'Is evolutionary theory realistic or anti-realistic?' In the traditional debate, the theory is anti-realistic if it will be recast with increasing our knowledge or information. If we follow this formulation, Fisher's formula of natural selection is interpreted as anti-realistic. For the formula of natural selection will vary with the amount of information. But this seems to miss the crucial feature of the formula. I argue that the formula of natural selection captures some aspects of reality. For example, Drosophila's colored eyes really inherit from ancestors and

${ }^{16}$ See Crow and Kimura (1970), and Morimoto and Nishiwaki (2006). 
information about this fact is considered in the formula of natural selection. From information theoretical point of view, we can understand that the formula of natural selection implies both epistemic and realistic factors. And this is the significant feature of the formula of natural selection that the traditional arguments can't grasp.

\section{Conclusion}

I have discussed the issue whether evolutionary theory is interpreted to be used the probability concept realistically or anti-realistically, by using Fisher's formula of natural selection as an example. The crucial point in my argument is that evolutionary theory including the formula of natural selection has the mixture of epistemic and realistic factors. Since this feature of evolutionary theory can't be grasped enough from the bipolarized points of view, the traditional arguments miss the point. For example, zebra's color is the mixture of white and black. If you are asked whether zebra is white or black, you will be troubled how to answer. And such a question is hardly meaningful to grasp the characters of zebra. If we want to know the characters of zebra, we should ask different types of questions. For the formula of natural selection, I have argued, full information or complete knowledge is not necessary. Like information theory, evolutionary theory can rationally predict or explain phenomena. In calling a theory rational here, we mean that it can be derived by means of utilizing partial information most efficiently. From information theoretical point of view, evolutionary theory can be interpreted as rational one. Thus if we hope to grasp the features of evolutionary theory, we should ask not whether it is realistic or anti-realistic, but rather whether it is rational or irrational. And to the latter question, I answer it is rational. Zebra has white and black striped pattern. So if we want to know the essential characters of zebra, we wouldn't ask whether it is white or black. Rather we would ask, for example, whether it is striped or not.

From information theoretical point of view, evolutionary theory can be interpreted as rational one. If this interpretation is sound, we can also understand the features of prediction and explanation in evolutionary theory. We will predict the change in gene frequency rationally if we utilize partial information, i.e. maximize functions. The more information we have, the more rational or accurate prediction we can make. And we can explain the effect of natural selection rationally if we manage to discard information depending on our purpose. Even if we had full information, we wouldn't use almost all of them in explanation. This means that the aim of evolutionary theory is rational prediction or explanation of phenomena on the basis of partial information, not complete description of it on the basis of full information. This is another significant feature of evolutionary theory that the traditional arguments also can't grasp.

Information theory gives us a rational principle. Jaynes applied maximum en- 
tropy principle to statistical mechanics. My argument in this paper owes much to his work. He claims, "we have now reached a state where statistical mechanics is no longer dependent on physical hypotheses, but may become merely an example of statistical inference." (1957a p.621) By statistical inference he means maximum entropy principle used in information theory. For him, statistical mechanics is a part of information theory. Moreover, the field of quantum information theory has recently developed dramatically. So some physicists and philosophers of physics reexamine quantum theory from information theoretical point of view. Philosophers of physics, Clifton, Bub and Halvorson, for example, claim as follows. "We therefore suggest substituting for the conceptually problematic mechanical perspective on quantum theory an information-theoretic perspective. That is, we are suggesting that quantum theory be viewed, not as first and foremost a mechanical theory of waves and particles (...), but as a theory about the possibilities and impossibilities of information transfer." (2003 p.1563) Whether statistical mechanics or quantum mechanics will become to be a part of information theory, it is certain that information theory will be regarded as important in science. In future it may come to be true that nothing in science makes sense except in the light of information.

\section{Appendixes}

\section{A1. Maximum Entropy Principle}

Let $x_{i}(i=1,2, \ldots n)$ stand for events or propositions. We are not given the corresponding probability $p_{i}$, but we know only the expectation of function $\bar{f}$

$$
\bar{f}=\sum_{i} p_{i} f_{i}
$$

and the axioms of probability

$$
\sum_{i} p_{i}=1
$$

In addition, Shannon proved that the quantity, which is positive, which increases with increasing uncertainty, and which is additive for independent source of uncertainty, is the information entropy function $H$

$$
H=-\sum_{i} p_{i} \log p_{i}
$$

In deriving $p_{i}$ on the basis of partial information, we ought to use the probability which has maximum entropy subject to whatever we know. We show that maximization of $H$ leads to probability $p_{i}$ by equating the derivation to 0 subject to some constraints. Maximizing $H$ yields

$$
\begin{aligned}
& d H=0 . \\
& -68-
\end{aligned}
$$


Now there are two constraints. The first is one of the axioms of probability (A1.2). Differentiating this gives

$$
\sum_{i} d p_{i}=0
$$

The second constraint is the expectation of $f$. Since it is constant, differentiating it becomes

$$
\sum_{i} f_{i} d p_{i}=0
$$

We maximize $H$ by the method of Lagrange multipliers. We let

$$
d H-\mu \sum_{i} d p_{i}-\lambda \sum_{i} f_{i} d p_{i}=0
$$

where $\lambda$ and $\mu$ are Lagrange multipliers. Now differentiating (A1.3) gives

$$
\frac{d H}{d p_{i}}=-\sum_{i} \frac{d p_{i} \log p_{i}}{d p_{i}}=-\sum_{i}\left(1+\log p_{i}\right) .
$$

Substituting this into (A1.7) yields

$$
-\sum_{i}\left[\left(1+\log p_{i}\right)+\mu+\lambda f_{i}\right] d p_{i}=0
$$

All these coefficients of $d p_{i}$ must be 0 in order to satisfy this identical equation. Then

$$
\left(1+\log p_{i}\right)+\mu+\lambda f_{i}=0
$$

Transforming this equation yields

$$
p_{i}=\exp \left(-\lambda_{0}-\lambda f_{i}\right)
$$

where $\lambda_{0} \equiv \mu+1$. Substituting this into (A1.2) becomes

$$
1=\sum_{i} p_{i}=\sum_{i} \exp \left(-\lambda_{0}-\lambda f_{i}\right)=\exp \left(-\lambda_{0}\right) \sum_{i} \exp \left(-\lambda f_{i}\right)
$$

Then

$$
\exp \left(\lambda_{0}\right)=\sum_{i} \exp \left(-\lambda f_{i}\right)
$$

Substituting this into (A1.11) gives

$$
p_{i}=\frac{\exp \left(-\lambda f_{i}\right)}{\sum_{i} \exp \left(-\lambda f_{i}\right)} .
$$

Thus probability $p_{i}$ can be derived from maximum entropy principle.

$$
-69-
$$




\section{A2. Maximum Principle of Natural Selection}

Fisher (1930) formulated Darwin's idea of natural selection mathematically and derived the formula of natural selection called, 'fundamental theorem of natural selection.' He postulates that (1) the same allele has an equal fitness, (2) there is an allele at a single locus, (3) the size of population is large, and (4) organisms mate randomly. According to Fisher's theorem of natural selection, when natural selection works at the population, the change of the average fitness $\bar{w}$ in the population is expressed

$$
\frac{d \bar{w}}{d t}=\sum p_{i}\left(w_{i}-\bar{w}\right)^{2}
$$

where $p_{i}$ is the frequency of the allele $i$ in the population and $w_{i}$ is the fitness of that allele. Fisher shows that the change of the average fitness equals to the variance of the population. His formula, however, doesn't explain or predict the change in gene frequency. So Kimura derived the change in gene frequency $p_{i}$ under the same conditions as Fisher's formula by the principle called, 'maximum principle of natural selection."

Let us consider a single locus with genes in random mating population. We can define the average fitness from the postulation of random mating

$$
\bar{w}=\sum_{i} p_{i} w_{i}
$$

Then for any set of small change in gene frequency $d p_{i}$, the change in the average fitness is

$$
d \bar{w}=\sum_{i, j} w_{i j} p_{j} d p_{i}+\sum_{i, j} w_{i j} p_{i} d p_{j}=2 \sum_{i} w_{i} d p_{i}
$$

We show that maximization of $d \bar{w}$ leads to equations of gene frequency change by equating the derivation to 0 subject to some constraints. Now there are two constraints. The first is one of the axioms of probability

$$
\sum_{i} p_{i}=1
$$

Differentiating this gives

$$
\sum_{i} d p_{i}=0
$$

The second constraint is that the genetic variance is constant. From the definition of fitness, ${ }^{17}$

$$
\frac{d p_{i}}{d t}=p_{i}\left(w_{i}-\bar{w}\right) .
$$

And by substituting it into Fisher's theorem of natural selection, (A2.1) becomes

\footnotetext{
${ }^{17}$ For details, see Kimura (1958), and Crow and Kimura (1970 section 5.3 and 5.9).
} 


$$
\frac{d \bar{w}}{d t}=2 \sum_{i} \frac{1}{p_{i}}\left(\frac{d p_{i}}{d t}\right)^{2}
$$

So we write as the second constraint

$$
\sum_{i} \frac{1}{p_{i}}\left(\frac{d p_{i}}{d t}\right)^{2}=c
$$

where $c$ is a constant, equal to $\Sigma p_{i}\left(w_{i}-\bar{w}\right)^{2}$.

We maximize $d \bar{w}$ by the method of Lagrange multipliers. We let

$$
L=d \bar{w}+\lambda\left(\sum_{i} \frac{\left(d p_{i}\right)^{2}}{d p_{i}}-c(d t)^{2}\right)+\mu\left(\sum_{i} d p_{i}\right),
$$

where $\lambda$ and $\mu$ are Lagrange multipliers. Differentiating this gives

$$
\frac{d L}{d p_{i}}=\frac{d \bar{w}}{d p_{i}}+\lambda \frac{d}{d\left(d p_{i}\right)}\left(\sum_{i} \frac{\left(d p_{i}\right)^{2}}{d p_{i}}-c\right)+\mu \frac{d}{d\left(d p_{i}\right)}\left(\sum_{i} d p_{i}\right)=0
$$

and equating $d L / d p_{i}$ to 0 gives

$$
2 w_{i}+2 \lambda \frac{d p_{i}}{p_{i}}+\mu=0
$$

We multiply each term by $p_{i}$ and sum this over all genes, $i=1,2, \ldots, n$.

$$
2 \sum_{i} w_{i} p_{i}+2 \lambda \sum_{i} d p_{i}+\mu \sum_{i} p_{i}=2 \bar{w}+\mu=0
$$

Thus

$$
\mu=-2 \bar{w} .
$$

Substituting it into (A2.11) yields

$$
2 w_{i} p_{i}+2 \lambda d p_{i}-2 \bar{w} p_{i}=0 .
$$

Thus

$$
d p_{i}=\frac{\left(w_{i}-\bar{w}\right) p_{i}}{-\lambda}
$$

Substituting it into (A2.8) becomes

$$
\sum_{i} \frac{\left(d p_{i}\right)^{2}}{p_{i}}=\sum_{i} \frac{\left(\frac{w_{i}-\bar{w}}{-\lambda} p_{i}\right)^{2}}{p_{i}}=\sum_{i} \frac{\left(w_{i}-\bar{w}\right)^{2} p_{i}}{\lambda^{2}}=c(d t)^{2} .
$$

Noting that $c$ is equal to $\Sigma p_{i}\left(w_{i}-\bar{w}\right)^{2}$ and we want fitter gene to increase in frequency, 
not decrease,

$$
\frac{1}{\lambda}=-d t
$$

Substituting it into (A2.15) gives

$$
\frac{d p_{i}}{d t}=p_{i}\left(w_{i}-\bar{w}\right)
$$

Thus the equations for the change of gene frequency can be derived from maximum principle of natural selection.

\section{References}

[1] Brandon, R. and Carson, S. (1996) "The Indeterminism Character of Evolutionary Theory: No 'No Hidden Variable Proof' but No Room for Determinism Either", Philosophy of Science 63: 315-337.

[2] Clifton, R., Bub, J. and Halvorson, H. (2003) "Characterizing Quantum Theory in Terms of Information-Theoretic Constraints", Foundations of Physics 33: 1561-1591.

[3] Crow, J. and Kimura, M. (1970) An Introduction to Population Genetics Theory, Harper and Row.

[4] Darwin, C. (1859) The Origin of Species, Harvard University Press, 1964.

[5] Dobzhansky, T (1973) "Nothing in Biology Makes Sense Except in the Light of Evolution", The American Biology Teacher 35: 125-129.

[6] Fisher, R. (1930) The Genetical Theory of Natural Selection, $2^{\text {nd }}$ ed., Dover.

[7] Glymour, B. (2001) "Selection, Indeterminism, and Evolutionary Theory", Philosophy of Science 68: 518-535.

[8] Graves, L., Horan, B. and Rosenberg, A. (1999) "Is Indeterminism the Source of the Statistical Character of Evolutionary Theory?", Philosophy of Science 66: 140-157.

[9] Horan, B. (1994) "The Statistical Character of Evolutionary Theory", Philosophy of Science 61: 76--95.

[10] Jaynes, E. (1957a) "Information Theory and Statistical Mechanics", Physical Review, 106: $620-630$.

[11] Jaynes, E. (1957b) "Information Theory and Statistical Mechanics II", Physical Review, 108: $171-190$.

[12] Jaynes, E. (1963) "Information Theory and Statistical Mechanics", in Statistical Physics, K Ford (ed.), W.A. Benjamin, Inc., 181-218.

[13] Jaynes, E. (2003) Probability Theory, Cambridge University Press.

[14] Kimura, M. (1958) "On the Change of Population Fitness by Natural Selection", Heredity 12: $145-167$.

[15] Lewontin, R. (1970) "The Units of Selection", Annual Review of Ecology and Systematic 
1: 1-14.

[16] Mayr, E. (1959) "Typological versus Population Thinking", in Evolution and Anthropology: A Centennial Appraisal, 409-412.

[17] Morimoto, R. (2007a) "Determinism and the Probability Concept: what is the evolutionary world view?" (in Japanese), The Heredity (suppl.) 20: 146-150.

[18] Morimoto, R. (2007b) "Irreducibility of Evolutionary Theory" (in Japanese), journal of the Japan Association for Philosophy of Science 40: 15-27.

[19] Morimoto, R. and Nishiwaki, Y. (2006) "Probabilistic Reasoning in Evolutionary Theory", Reasoning and Cognition Interdisciplinary Conference Series on Reasoning Studies 2: $181-185$.

[20] Price, G.R. (1970) "Selection and Covariance", Nature 227: 520-521.

[21] Rosenberg, A. (1994) Instrumental Biology, or The Disunity of Science, The University of Chicago Press.

[22] Roughgarden, J. (1979) Theory of Population Genetics and Evolutionary Ecology, Prentice-Hall.

[23] Shannon, C. (1948) "A Mathematical Theory of Communication", Bell System Technical Journal 27: 379-423.

[24] Sober, E. (1984) The Nature of Selection, The MIT Press.

[25] Sober, E. (1993) Philosophy of biology, Oxford University Press.

[26] Stamos, D. (2000) "Quantum Indeterminism and Evolutionary Biology", Philosophy of Science 68: 164-184.

[27] Wright, S. (1931) "Evolution in Mendelian Populations", Gnenetics 16: 97-159. 